\title{
IDENTIDADE DE GÊNERO/SEXO DE ATLETAS E SEDENTÁRIOS!
}

\author{
PH.D. FERNANDO LUIZ CARDOSO
}

Ph.D Professor de Educação Física do Programa de Pós-Graduação em Ciências do Movimento Humano da Universidade do Estado de Santa Catarina - UDESC (Florianópolis - Santa Catarina Brasil, Coordenador do Laboratório de Gênero, Sexualidade e Corporeidade (LAGESC) do Centro de Saúde e Esportes da UDESC

E-mail: fernandocardoso.ph.d.lagesc@gmail.com,

\section{MS. CINARA SACOMORI}

Fisioterapeuta, doutoranda em Ciências do Movimento Humano junto a

Universidade do Estado de Santa Catarina - UDESC

(Florianópolis - Santa Catarina - Brasil)

E-mail: cinarasacomori@gmail.com

\begin{abstract}
RESUMO
Existem diferenças no processo de estruturação da identidade de gênero/sexo de atletas e não-atletas? Este estudo teve como objetivo comparar a identidade de gênero/sexo autopercebida e autoquantificada de atletas de diferentes modalidades (I 80 homens e 180 mulheres) em relação a sedentários (22 homens e 22 mulheres), no tocante a personalidade e preferências lúdicas da infância. Os homens se perceberam mais masculinos e as mulheres mais femininas. Encontraram-se mais mulheres não heterossexuais do que homens, entre as atletas. Os homens assumiram-se mais propensos a gostar de quebrar as regras e sonhavam em ser campeão esportivo quando crianças. As atletas mulheres que brincaram mais com meninos na infância se avaliaram como mais masculinas, ousando em transgredir as fronteiras dos papéis de gênero, o que pode ter facilitado o interesse delas pelo treinamento esportivo.
\end{abstract}

PALAVRAS-CHAVES: Esporte; identidade de gênero; masculinidade; feminilidade.

I. Este trabalho teve o apoio de uma bolsa de Iniciação a Pesquisa (PROBIC) fomentada pela Universidade do Estado de Santa Catarina entre 2009 e 2010. 
Nos meios acadêmicos, o termo gênero começou a ser utilizado na década de 70 como um conceito que indica a rejeição ao determinismo biológico implícito no uso de termos como sexo ou diferenciação sexual. Desde então, o mundo acadêmico adquiriu uma nova noção relacional de análise para estudar os papéis sociais de homens e mulheres. (SCOTT, 1990). Diferente dessa matriz teórica, Money ( 1988) propôs o conceito de identidade de gênero/papel (IG/P) como um conceito integrante que não dissocia a relação natureza e cultura, que define o ser a partir de categorias como macho/fêmea ou intersexo (sexo biológico), masculino/ feminino ou andrógino (orientação de gênero), bissexual ou monossexual (orientação sexual) no âmbito pessoal, social e legal.

Compreende-se que homens e mulheres, em cada cultura, apresentam diferentes níveis de identificação com os tradicionais papéis sexuais. A maioria dos indivíduos estrutura sua orientação de gênero de forma convergente com o seu sexo biológico, enquanto outros podem apresentar diferentes níveis de congruência. (CARDOSO, 1994). Além disso, essa convergência ou divergência não é absoluta, pois pode ocorrer em diferentes níveis, por exemplo, uma mulher pode ter características muito femininas em sua cultura, mas possuir uma capacidade de liderança muito proeminente que acabe masculinizando o seu comportamento ou a percepção que os outros têm dela. Ou ainda, homens e mulheres podem apresentar características intermediárias em termos de personalidade, atitudes, indumentária e motricidade, que serão designadas de andróginas. Desse modo, os masculinos podem ser definidos como sendo aqueles indivíduos cuja masculinidade predomina sobre a feminilidade, sendo o contrário atribuído aos indivíduos mais femininos. (SOARES; ASSIS; SOUSA, 2005). Nesse sentido, a orientação de gênero do presente artigo segue o conceito de Donelson e Gullahorn, (1977), Wakefield et al. (1976) e Money (1988), que o definem como a variabilidade gradual dos níveis de androgenia de um indivíduo entre os extremos de masculinidade e feminilidade em uma escala contínua.

Atividades denominadas de masculinas ou femininas são produto de uma construção social e histórica, e os esportes e as atividades físicas sofrem essa mesma interferência dos estereótipos de gênero. (KOIVULA, 1995). A maioria dos esportes populares são percebidos como masculinos, mas esportes com características eminentemente femininas também existem e geralmente estão mais próximos das artes e da estética. Koivula (200 I) investigou como homens e mulheres atletas percebem os diferentes esportes em termos de adequabilidade aos tradicionais papéis de gênero, e descobriu que essa percepção nativa poderia ser subdividida 
em esportes: neutros, femininos e masculinos, levando em consideração muitos aspectos como estética e feminilidade, perigo e risco, velocidade e masculinidade.

Esportes competitivos geralmente são percebidos como ícones de masculinidade em muitas sociedades. (SNYDER; SPREITZER, I983; CZIMA; WITTIG; SCHURR, 1988; ECCLES; HAROLD, 199I; JAFFEE; MANZER, 1992; KOIVULA, 1995, 1999, 200I; MARTIN; MARTIN, 1995; SHAW; KLEIBER; CALDWELL, 1995; MILLER; LEVY, 1996; GIBBONS; LYNN, 1997; CARDOSO, 2009). Homens que não praticam esportes são socialmente percebidos como tendo algum desvio de identidade em termos de orientação de gênero ou orientação sexual. (GREEN, 1987; MESSNER; SABO, 1994; CARDOSO, 2009). Por outro lado, mulheres empenhadas na prática de esportes considerados masculinos recebem similar percepção de desvio. (BEM, 1974; COLLEY; ROBERTS; CHIPPS, 1985; SABO, 1985; MESSNER; SABO, 1994; BRADY et al., 1996; ECCLES et al., 1999; HARRISON; LYNCH, 2005).

A categorização da orientação de gênero dos esportes criada por Koivula (200 I) ainda não foi testada no Brasil. Kamizake (20 I 0) produziu, de forma indireta, a partir da preferência esportiva de estudantes universitários de ambos os sexos, dados que podem ser usados para apontar a autopercepção de gênero dos brasileiros. Cardoso et al. (2009) e Cardoso et al. (20 I0) produziram os primeiros dados quantitativos sobre a identidade gênero/sexo entre homens e mulheres atletas e de praticantes de dança no Brasil, respectivamente.

A Revista Brasileira de Ciências do Esporte, em seu volume 15 de Janeiro de 1994, introduziu a problematização dos estereótipos de gênero e dos papéis sociais adequados a homens e mulheres e as suas possíveis influências na Educação Física brasileira, e contou com vários colaboradores naquele intento: Romero (1994) discutiu o aprendizado dos tradicionais papéis sexuais na família e a sua reificação na Educação Física escolar; Taffarel e França ( 1994) discutiram a participação da mulher nos Jogos Olímpicos e na produção do conhecimento científico na Educação Física; Saraiva-Kunz ( 1994) discutiu o sexismo e as possibilidades de superação deste nas aulas de Educação Física; Chagas ( 1994 ) discutiu a padronização do corpo feminino pela ideologia dominante; Soares e Goellner (1994) discutiram a marginalização da diferença, Cardoso ( 1994 ) propôs um repensar das aulas de Educação Física a partir de um projeto coeducativo que respeite a relação natureza e cultura.

Na década passada a RBCE passou a receber outras contribuições que já assumiam uma nova noção relacional de análise, que propunha uma separação entre sexo biológico e identidade, com ênfase nas construções sociais simbólicas proposta por Scott ( 1990). Costa e Silva (2002) discutiram a temática no tocante à diferença entre as aulas mistas e as aulas co-educativas pautadas pelo princípio da 
igualdade e diferença. Lucena (2003) utilizou-se de uma análise histórica das crônicas no Brasil para resgatar as primeiras ações esportivas. Damico e Meyer (2006) discutiram alguns dos modos pelos quais jovens mulheres significam, aprendem e vivenciam o cuidado com o corpo. Humberstone (2007) examinou os diversos sentidos filosóficos atribuídos à natureza, e chama a atenção para o predomínio de vozes masculinas nos discursos acadêmicos que se preocupam com a natureza e as atividades ao ar livre. Melo e Lacerda (2009) discutiram as relações entre esporte e dança, que de alguma forma expressavam representações relacionadas ao quadro de tensões do contexto sociocultural no século $X X$, entre as quais estão as questões de gênero. Desde então, outros pesquisadores têm dado suas contribuições na mesma abordagem epistemológica. No entanto, pouco se tem produzido no âmbito da Educação Física nacional que avance para além da problematização ou difusão do construto de gênero difundido por Scott (1990), que promove uma separação de sexo e gênero. Sob outro ponto de vista (MONEY, 1988), esse artigo aborda o gênero baseado em outra definição do mesmo construto, a qual integra natureza e cultura no conceito de identidade de gênero/sexo. Baseado na escala de Kinsey (1948), que mensurava orientação sexual, Cardoso (2008) criou uma escala equivalente para mensurar a identidade de gênero.

Assim, percebe-se que a maioria das publicações nacionais tem forte influência de métodos interpretativos e de cunho mais simbólicos. Infelizmente não se está produzindo no Brasil pesquisas mais explicativas, isto é, que se preocupem em explorar uma relação de causalidade sobre o fenômeno do gênero articulado ao conceito de identidade de gênero/sexo (MONEY, 1988), pautadas em dados atuais de campo que nos ajudem não só a compreender as dinâmicas das relações de gênero no contexto atual do esporte e da escola, mas também, explicá-las.

A partir das contribuições de alguns poucos autores internacionais aqui citados, dentre outros que produziram alguns dados quantitativos para tentar uma graduação científica da orientação de gênero em pessoas com diferentes níveis de contato com os esportes, questiona-se: Existem diferenças no processo de estruturação da identidade de gênero/sexo entre atletas e não-atletas? Este estudo teve como objetivo comparar a identidade de gênero/sexo autopercebida e autoquantificada entre atletas e sedentários, controlando o sexo biológico.

\section{MÉTODOS}

Trata-se de uma pesquisa de campo de natureza descritivo-comparativa entre grupos, pois se propõe analisar as diferenças e as associações entre as variáveis, com o objetivo de comparar a identidade autopercebida de gênero/sexo de homens e mulheres atletas praticantes de várias modalidades esportivas e sedentários. 
Esta pesquisa utilizou a auto-avaliação por intermédio do questionário - Questionário de Identidade Corporal (QIC), constituído de três dimensões (sexualidade, corporeidade e motricidade), além dos itens: aspectos gerais e questões sociais. No início do questionário, abordam-se aspectos gerais do pesquisado como: a idade, profissão, estado civil, sexo biológico, gênero e orientação sexual; e questões sociais: que são os bens de consumo que o pesquisado possui em casa, o grau de escolaridade e a prática de atividade física. $\bigcirc$ questionário é composto por nove escalas: intimidade corporal, percepção corporal, satisfação corporal, identidade infantil, pré-disposição sexual, comportamento sexual, orientação sexual, satisfação sexual, experiência motora e orientação motora. Cada escala é medida através de uma Escala Likert de sete níveis (nunca - médio - muito, quantificados da seguinte forma: $0-1-2-3-4-5-6$ ). O referido instrumento baseou-se na Escala Kinsey (KINSEY et al., 1948) que foi criado para graduar a orientação sexual humana. Atualmente o QIC encontra-se em fase final de validação, possuindo autorização para ser utilizado pelo Comitê de Ética em Pesquisa com Seres Humanos da Universidade do Estado de Santa Catarina, com o número de Referência 40/05.

\section{PARTICIPANTES}

Os participantes da pesquisa foram 360 ( 180 homens e I 80 mulheres) atletas de ambos os sexos, com mais de 18 anos de idade, das seguintes modalidades esportivas: natação, voleibol, basquetebol, handebol e futebol, entre os meses de Março a Junho de 2009. Paralelamente, aplicou-se o mesmo instrumento com 44 indivíduos sedentários de ambos os sexos na mesma faixa etária.

Em termos de perfil sócio-antropométrico, os homens foram mais ativos fisicamente e tiveram o início da suas vidas sexuais mais cedo do que as mulheres. Os homens atletas tiveram a sua iniciação sexual significativamente mais precoce do que as mulheres atletas. Não existe diferença significativa em termos de idade, bens de consumo e estado civil entre os sexos, porém os homens sedentários têm uma escolaridade ligeiramente menor. As mulheres, como esperado, avaliaram-se como mais femininas em termos de orientação de gênero e assumiram uma orientação sexual predominantemente heterossexual, em que as mulheres atletas apresentaram, significativamente, maior média na escala de orientação sexual (0 representando totalmente heterossexual e 6 totalmente homossexual) do que os outros grupos. Detalhes na Tabela I e nas Figuras I e 2. 
Tabela I. Perfil Sócio-antropométrico dos participantes atletas e grupo controle

\begin{tabular}{|c|c|c|c|c|c|c|c|c|c|c|}
\hline \multirow[t]{3}{*}{ Parâmetros } & \multicolumn{2}{|c|}{ Homens $(\mathrm{N}=180)$} & \multicolumn{2}{|c|}{$\begin{array}{l}\text { Mulheres } \\
(\mathrm{N}=\mid 80)\end{array}$} & \multicolumn{2}{|c|}{$\begin{array}{l}\text { Homens } \\
(\mathrm{N}=22)\end{array}$} & \multicolumn{2}{|c|}{$\begin{array}{l}\text { Mulheres } \\
(\mathrm{N}=22)\end{array}$} & \multirow[t]{2}{*}{$\mathrm{F}$} & \multirow[t]{2}{*}{$p$} \\
\hline & $x$ & \pm & $x$ & \pm & $x$ & \pm & $x$ & \pm & & \\
\hline & \multicolumn{4}{|c|}{ Atleta } & \multicolumn{4}{|c|}{ Grupo Controle } & & \\
\hline Idade (anos) & 21.76 & 3.27 & 21.41 & 3.28 & 22.50 & 2.22 & 21.23 & 1.60 & 1.015 & n.s \\
\hline Altura (cm) & $1.80^{b}$ & .06 & $1.65^{\mathrm{a}}$ & .06 & $1.77^{\circ}$ & .07 & $1.66^{\mathrm{a}}$ & .06 & $|35.14|$ & .001 \\
\hline Peso (kg) & $76.80^{b}$ & 9.08 & $60.0^{\mathrm{a}}$ & 8.16 & $76.25^{b}$ & 13.23 & $61.45^{\mathrm{a}}$ & 9.60 & 100.815 & .001 \\
\hline IMC & $23.57^{b}$ & 2.41 & $21.83^{\mathrm{a}}$ & 2.64 & $24.00^{b}$ & 3.78 & $22.19^{\mathrm{a}}$ & 2.89 & 13.206 & .001 \\
\hline $\begin{array}{l}\text { Idade da la experiência } \\
\text { sexual (anos) }\end{array}$ & $15.95^{\mathrm{a}}$ & 1.62 & $17.09^{\mathrm{b}}$ & 1.95 & 16.35 & 2.36 & 16.63 & 2.11 & 9.730 & .001 \\
\hline Orientação de Genero' & $.47^{\mathrm{a}}$ & .75 & $5.06^{b}$ & 1.00 & $.41^{\mathrm{a}}$ & .59 & $5.23^{\circ}$ & .68 & 860.285 & .001 \\
\hline Orientação sexual $\left.\right|^{2}$ & $.11^{\mathrm{a}}$ & .55 & $.57^{\circ}$ & 1.38 & $.18^{\mathrm{a}}$ & .39 & $.23^{\mathrm{a}}$ & .42 & 5.736 & .001 \\
\hline Itens de comsumo ${ }^{3}$ & 4.09 & 1.21 & 4.01 & 1.20 & 3.68 & 1.17 & 4.41 & 1.05 & 1.483 & n.s \\
\hline Escolaridade $^{4}$ & $4.95^{b}$ & .51 & $4.95^{b}$ & .56 & $4.55^{a}$ & 1.14 & $4.95^{b}$ & .37 & 3.342 & .01 \\
\hline \multirow[t]{2}{*}{ Nível de atividade fisica ${ }^{5}$} & $4,24^{b}$ & 1.30 & $3.64^{b}$ & 1.50 & $1.29^{\mathrm{a}}$ & 1.27 & $1.05^{\mathrm{a}}$ & .95 & 56.146 & .001 \\
\hline & $\mathrm{N}$ & $\%$ & $N$ & $\%$ & $\mathrm{~N}$ & $\%$ & $\mathrm{~N}$ & $\%$ & & $p$ \\
\hline Casado ou cônjuge 6 & 14 & 8.50 & 12 & 7.40 & 2 & 4.5 & 3 & 7.8 & 1.45 & n.s \\
\hline
\end{tabular}

Observação: Sig. (2-tailed) $\leq .05$ para teste de One way ANOVA

I. Questão: Gênero (como você se percebe em termos de identidade corporal?): (0 muito masculino e 6 muito feminino). Media aritmética de uma escala tipo Likert (0- Muito Masculino, I- Masculino, 2- Mediano, 3- Mediano, 4- Mediano, 5- Feminino, 6- Muito Feminino).

2. Questão: Orientação sexual (Como você definiria a sua preferência sexual?): (0 totalmente heterossexual e 6 totalmente homossexual). Media aritmética de uma escala tipo Likert (0- Totalmente heterossexual, I - Heterossexual, 2- Bissexual, 3- Bissexual, 4- Bissexual, 5- Homossexual, 6- Homossexual).

3. Questão: Quais destes itens de consumo você tem em sua casa? ( ) TV, ( ) TV a cabo, ( ) Computador, ( ) Internet, ( ) Ar condicionado, ( ) Carro. Media aritmética do que assumiu possuir em casa.

4. Questão: Qual e o seu nível de escolaridade? 0. ( ) Nenhuma educação formal, I. ( ) I Grau incompleto, 5. () I Grau completo, 3. ( ) II Grau incompleto, 4. ( ) II Grau completo, 5. ( ) Graduação, 6. ( ) Pós-graduação. Media aritmética da escolaridade assumida.

5. Questão: $O$ quanto ativo você se considera em termos de atividade física? Media aritmética de uma escala tipo Likert (0- Nada, I- Pouco, 2- Razoável, 3- Mediano, 4-Satisfatório, 5- Bem, 6- Muito).

6. Questão: Qual é o seu estado co-habitacional? ( ) solteiro sem nenhum relacionamento amoroso, ( ) solteiro com um relacionamento amoroso, ( ) mora com o parceiro-a ou cônjuge, ( ) separado ou divorciado ou viúvo e vive só, ( ) separado ou divorciado ou viúvo e vive com alguém. Percentual de participantes que são casados ou vivem com parceiro-a ou cônjuge.

Os 44 participantes sedentários que serviram de grupo controle têm similar características em termos de idade, origem social e medidas antropométricas. Porém os homens atletas apresentaram um nível de escolaridade ligeiramente mais alto do que o grupo controle e as mulheres em geral. A função deste grupo é ajudar a delimitar e contextualizar as fronteiras de influência do esporte sobre os atletas participantes, bem como a influência do esporte sobre a personalidade e preferências lúdicas ou vice-versa. 


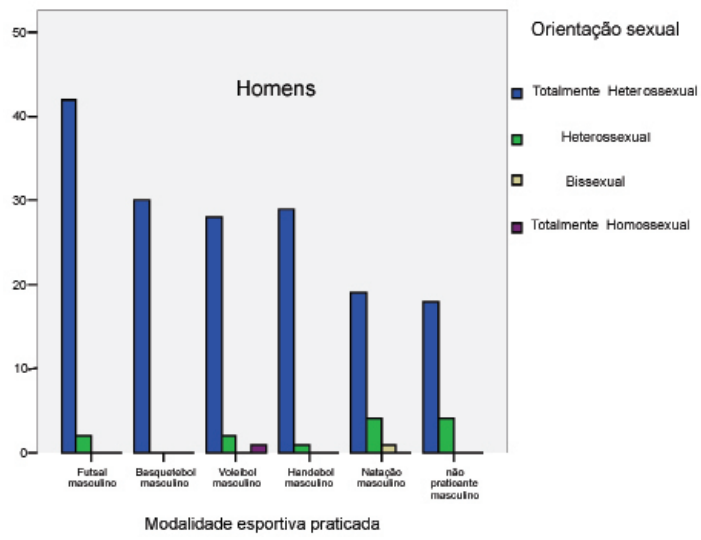

Figura I. Distribuição da orientação sexual (0, I-homossexual;

2, 3, 4-bissexual; 5, 6-heterossexual) dos participantes homens da pesquisa.

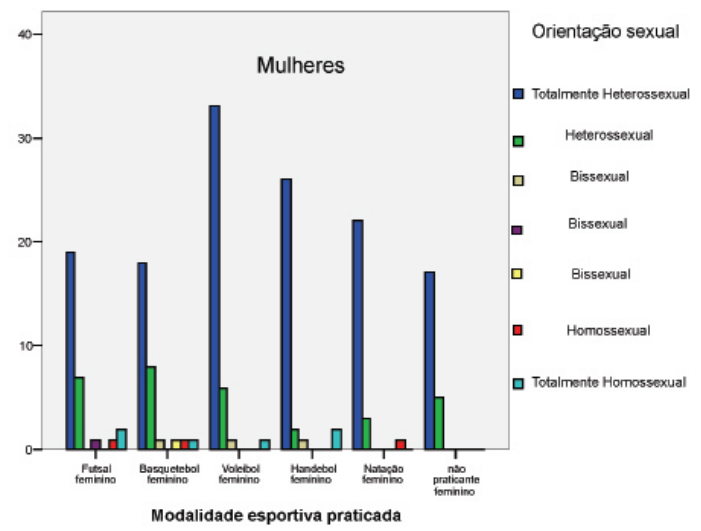

Figura 2. Distribuição da orientação sexual (0, I-homossexual; 2, 3, 4-bissexual; 5, 6-heterossexual) das participantes mulheres da pesquisa.

As mulheres, na sua maioria, se autoavaliaram como heterossexuais, no entanto, apresentaram maior diversidade ou heterogeneidade em termos de orientação sexual. Maiores detalhes sobre a incidência de bissexuais e homossexuais entre as mulheres por grupo encontram-se na Figura 2.

\section{PROCEDIMENTOS}

Os dados foram coletados via técnica snow ball e focus group em universidades, escolas, ginásios esportivos, e nos locais oficiais de competição. Ambos os grupos 
receberam um questionário em branco que foi preenchido individualmente, depois dobrado, envelopado e depositado em uma urna lacrada, que foi aberta apenas no final de cada coleta com o objetivo de proteger o anonimato dos participantes, de acordo com a aprovação do Comitê de Ética em Pesquisa com Seres Humanos, n 40/50, resolução CNS 196/96. Os dados foram coletados entre Março e Junho de 2009.

Os dados foram organizados e analisados no Programa Estatístico SPSS versão 17.0, através de estatística descritiva (média, desvio-padrão e frequência) e por estatística inferencial, usando-se o Student's $t$ tests, One-Way analysis of variance (ANOVA) e o chi-squared test.

\section{RESULTADOS}

Identidade de gênero e orientação sexual dos atletas em relação ao grupo controle

Em relação à orientação de gênero, os homens se percebem mais masculinos e as mulheres mais femininas ( $t=-52.390, p \geq .001)$. Em relação à orientação sexual, encontraram-se mais mulheres não heterossexuais $(x=.57-+0-6, \pm$ I.37) do que homens $(x=.11-+0-6, \pm .54$ e $t=-4.021, p \geq .001)$ entre os atletas. Tal diferença não existe no grupo controle. As mulheres atletas ainda são mais heterogêneas em termos de orientação sexual, ou seja, variam mais entre a heterossexualidade, bissexualidade e a homossexualidade do que as mulheres do grupo controle $\left(X^{2}=25.734, p \geq .001\right)$.

A proporção de bissexuais e homossexuais em cada esporte está ilustrada na Tabela 2. Como se percebe, existe um maior número de bissexuais e homossexuais entre atletas mulheres nos diferentes esportes.

Tabela 2. Percentual de bissexualidade e homossexualidade entre os participantes por modalidade esportiva controlado pelo sexo

\begin{tabular}{lcccc}
\hline Esportes & \multicolumn{2}{c}{ Homens $(\mathrm{N}=180)$} & \multicolumn{2}{c}{ Mulheres $(\mathrm{N}=180)$} \\
\cline { 2 - 5 } & $\mathrm{n}-\mathrm{N}$ & $\%$ & $\mathrm{n}-\mathrm{N}$ & $\%$ \\
\cline { 2 - 5 } Natação & $\mathrm{I}-30$ & $3.3 \%$ & $1-31$ & $3.2 \%$ \\
Voleibol & $0-31$ & $0 \%$ & $2-41$ & $4.4 \%$ \\
Basquetebol & $0-30$ & $0 \%$ & $4-30$ & $13.2 \%$ \\
Handebol & $0-30$ & $0 \%$ & $3-31$ & $9.7 \%$ \\
Futebol & $0-44$ & $0 \%$ & $4-30$ & $13.2 \%$ \\
Grupo Controle & $0-22$ & $0 \%$ & $0-22$ & $0 \%$ \\
\hline & $X^{2}$ & $\mathrm{P}$ & $X^{2}$ & $\mathrm{P}$ \\
\hline & 14.795 & $\mathrm{n} . \mathrm{s}$ & 22.138 & $\mathrm{n} . \mathrm{s}$ \\
\hline
\end{tabular}

Observação: Sig. (2-tailed) $\leq .05$ 
Apesar de existir uma forte correlação entre as variáveis orientação de gênero e sexo biológico ( $r=.937, p \geq .00 \mathrm{I})$, ficou claro que alguns participantes ( $16 \%)$ se autoavaliaram como indivíduos mais andróginos (TABELA 3). A correlação permanece significativa para atletas $(r=.933, p \geq .00 \mathrm{I})$ e sedentários $(r=.968, p \geq .00 \mathrm{I})$, porém torna-se ligeiramente mais alta para os sedentários, o que indica uma maior congruência entre esses dois aspectos para os sedentários.

Tabela 3. Percentual dos participantes de ambos os sexos na categoria sexo biológico e na categoria orientação de gênero

\begin{tabular}{lccccc}
\hline & \multicolumn{3}{c}{ Sexo Biológico } & \multicolumn{3}{c}{ Orientação de Gênero } \\
\cline { 2 - 5 } & Homem & Mulher & Masculino & Andrógino & Feminino \\
\hline Freqüência & 180 & 180 & 164 & 58 & 138 \\
$\%$ & 50 & 50 & 45.4 & 16.4 & 38.2 \\
Total & 360 & & & 360 & \\
\hline
\end{tabular}

Como se percebe, os estereótipos sociais de gênero também afetam o mundo esportivo, em geral os participantes homens se sentem mais masculinos $(x=.46-+0-3, \pm .74)$ e as mulheres $(x=5.06-+0-6, \pm .98)$ mais femininas. Não se encontrou diferenças significativas entre atletas e grupo controle como um todo.

\section{PERSONALIDADE E PREFERÊNCIAS LÚDICAS NA INFÂNCIA}

Com o objetivo de explicar a variação de avaliação da identidade de gênero/ sexo entre homens e mulheres, alguns aspectos da personalidade e das preferências lúdicas foram comparados e diferenças significativas foram encontradas: os homens assumiram-se mais propensos a gostar de praticar esportes, gostar de brincar de luta e videogame, gostar de brigar fisicamente e verbalmente, gostar de quebrar as regras e sonhavam em ser um campeão esportivo quando crianças. Os homens também brincavam mais com outros do mesmo sexo e preferiam atividades mais típicas de meninos. Já as atletas mulheres geralmente brincavam mais na infância com outras meninas e gostavam mais das atividades desenvolvidas por elas. Entretanto, aquelas que brincaram mais com meninos $(r=-.200, p \geq .001)$ e aquelas que brincaram com brincadeiras típicas dos meninos $(r=-.208, p \geq .001)$, se avaliaram como mais masculinas. Maiores detalhes na Tabela 4. 
Tabela 4. Traços da personalidade e das preferências lúdicas de atletas homens e mulheres

\begin{tabular}{lccccccc}
\hline Variáveis & \multicolumn{2}{c}{ Homens } & \multicolumn{2}{c}{ Mulheres } & \\
\cline { 2 - 5 } & \multicolumn{2}{c}{$x$} & \pm & $x$ & \pm & T test & \multicolumn{1}{c}{ P } \\
\cline { 2 - 5 } Você gostava de praticar esportes? & 5.33 & 1.22 & 4.95 & 1.60 & 2.54 & .01 \\
Você gostava de brincar de lutas? & 3.85 & 1.68 & 2.49 & 1.99 & 6.98 & .001 \\
Você gostava de brincar com videogame? & 4.45 & 1.66 & 2.61 & 2.00 & 9.45 & .001 \\
Você gostava de brigar fisicamente? & 2.03 & 1.87 & 1.09 & 1.50 & 5.23 & .001 \\
Você gostava de brigar oralmente? & 2.17 & 1.71 & 1.58 & 1.70 & 3.25 & .001 \\
Você gostava de quebrar as regras? & 2.95 & 1.70 & 2.09 & 1.77 & 4.96 & .001 \\
Você sonhava em ser um campeão esportivo? & 4.71 & 1.65 & 4.18 & 2.00 & 2.73 & .007 \\
Você brincava com bonecas? & 4.74 & 1.16 & 4.28 & 1.44 & 3.31 & .001 \\
Você brincava com brincadeiras típicas de meninos? & 5.34 & 1.08 & 3.60 & 1.64 & 11.85 & .001 \\
Você brincava com as meninas? & 3.03 & 1.47 & 4.31 & 1.28 & -8.78 & .001 \\
Você brincava com brincadeiras típicas de meninas? & .91 & 1.17 & 4.08 & 1.42 & -23.10 & .001 \\
\hline
\end{tabular}

Observação: Sig. (2-tailed) $\leq .05$

As respostas acima variaram de acordo com a média aritmética de uma escala tipo Likert: (0- Nada, I- Pouco, 2- Razoável, 3- Mediano, 4- Satisfatório, 5- Bem, 6- Muito).

\section{DISCUSSÃO}

Orientação de gênero e orientação sexual dos atletas em relação ao grupo controle

Como esperado, os homens se perceberam mais masculinos e as mulheres mais femininas, dados também encontrados por Koivula ( 1999) e Koca, Asçı, e Kirazcl (2005) ao avaliarem atletas de ambos os sexos na Turquia e Suécia, respectivamente. Com relação à orientação sexual, pôde-se verificar que existem mais homossexuais ou bissexuais entre as mulheres atletas, como já indicavam os dados de LamontMills (200I), diferentemente do que ocorreu nos estudos com bailarinos de Bailey e Oberschneider (1997) e Cardoso et al. (2010), em que a maior diversidade de orientação sexual foi percebida entre os praticantes homens.

grupo controle das mulheres não se diferenciou das mulheres atletas em termos da autoavaliação de orientação de gênero, o que vai de encontro com outros estudos (COLKER; WIDOM, I 980; GILENSTAM; KARP; HENRIKSSON-LARSÉN, 2008), que talvez tenham avaliado atletas oriundas de esportes considerados socialmente mais masculinos.

Apesar de existir uma forte correlação entre sexo biológico e a uma orientação de gênero mais feminina ou mais masculina, percebeu-se que houve discrepância significativa entre essas duas categorias na autoavaliação da identidade dos 
participantes de ambos os sexos. Koivula (1999) e Koca, Asçı, e Kirazcı (2005), que aplicaram o Bem Sex Role Inventory em seus países, também encontraram essa discrepância e graduação entre masculino e feminino para ambos os sexos, de forma que nem todas as mulheres se avaliaram como absolutamente femininas, o mesmo acontecendo com os homens. Esse dado exemplifica a proposta de Scott (1990), que tenta desnaturalizar ou relativizar o conceito de orientação de gênero. Porém, ainda assim não nos permite desconectar sexo biológico e orientação de gênero.

Personalidade e preferências lúdicas na infância

Para Anderson (2009), os esportes de competição constituem uma instituição social organizada para definir certos padrões de masculinidade, impregnados desde a infância dos praticantes. Isso pode ter se refletido nos estudos de Bem (1974) e Sabo (1985), nos quais as meninas possuem dificuldade de conciliar a natureza competitiva com a sua feminilidade. Da mesma forma, Eccles et al. (1999) e Harrison e Lynch (2005) relatam que muitas jovens mulheres ainda acreditam existir um conflito inerente entre valores e preferências femininas com a grande competitividade que algumas atividades físicas proporcionam, características predominantemente masculinas. Tal situação se confirma, neste estudo, com um menor interesse das mulheres atletas participantes, pela prática do esporte na infância.

Os homens também brincavam mais com outros do mesmo sexo e preferiam atividades mais típicas de meninos. Já as atletas mulheres geralmente brincavam mais na infância com outras meninas e gostavam mais das atividades desenvolvidas por elas. Estes se constituem papéis sociais de gênero muito bem relatados por outros autores. (CONNOR; SERBIAN, 1977; LISS, 198I; PORMELEAU et al., 1990; ALEXANDER, 2003). As mulheres mais andróginas tiveram uma infância menos envolvida com tarefas típicas de meninas ou com meninas na infância, confirmando os dados de Miller Lite Report ( 1985 ) e Giuliano, Popp e Knight (2000), o que poderia explicar o porquê das mulheres mais andróginas darem mais valor à competição na prática esportiva do que as mulheres típicas nos estudos de Koivula ( 1999). Toyama (1977) e Greendorfer (1993) confirmam que meninas que brincaram com jogos ou brinquedos "típicos de meninos" têm maior probabilidade de se tornarem mulheres atletas. Whitam (1983) usa esses mesmos referenciais também para caracterizar a infância de mulheres homossexuais.

Todavia, os homens atletas participantes eram mais propensos a gostar de praticar esportes, confirmando os estudos de Koivula ( 1999) e Slavica (20 I 0), gostar de brincar de luta e videogame, confirmando os estudos de Colwell, Grady e Rhaki ( 1995) e Bryce e Rutter (2006), gostar de brigar fisicamente e verbalmente, o que confirma os dados de Rubenstein et al. (1987), gostar de quebrar as regras, como percebeu Koivula (1999) e sonhavam em ser um campeão esportivo, como 
identificou Whitam (1983). Além disso, os atletas de forma geral tiveram escores significativamente maiores no tocante a gostar de esportes de aventura e serem mais fisicamente ativos, como encontrou também Koivula (1999).

Assim, aulas de Educação Física coeducativas, quando bem planejadas, podem dar acesso a meninos e meninas a um universo de experiências motoras mais genéricas, relativizando as tradicionais fronteiras da identidade de gênero/sexo.

\section{CONCLUSÃO}

Homens e mulheres atletas e não-atletas sofrem as mesmas pressões sociais dos tradicionais estereótipos de gênero na estruturação da sua identidade de gênero/sexo, uma vez que sofrem a influência das tradicionais fronteiras entre o que é adequado ou não para cada sexo. No entanto, as mulheres atletas parecem ter sido mais autoconfiantes em transgredir algumas dessas fronteiras - como brincar mais com o sexo oposto e com as atividades típicas de meninos em suas infâncias - o que pode ter aberto a estas mulheres a possibilidade de se interessarem pela prática e treinamento esportivo.

Com este estudo descritivo quantitativo que ousa avaliar um fenômeno tão subjetivo como a identidade de genro/sexo e que contou com uma grande maioria de atletas vinculados a modalidades coletivas, não se pode afirmar uma relação causal direta da prática esportiva sobre a personalidade e as preferências lúdicas de seus praticantes. Mesmo porque a relação pode ser invertida, isto é, pessoas com certos traços psicológicos acabariam escolhendo atividades mais ou menos esportivas, ou mais ou menos competitivas. Entretanto, podemos confirmar uma relação muito forte entre orientação esportiva ou lúdica com as experiências motoras e sociais com o sexo oposto na infância para as mulheres. Infelizmente, a seleção das modalidades esportivas e dos atletas foi por disponibilidade. Sugere-se que próximos estudos usem um critério mais rigoroso de seleção e classificação das modalidades esportivas a partir de sua natureza.

Os dados nos mostram, também, que não existe uma relação direta entre orientação de gênero (masculino, andrógino, feminino) e orientação sexual (heterossexualidade, bissexualidade, homossexualidade), mas sim uma relação entre identidade de gênero/sexo e orientação esportiva (esportes mais competitivos, ou mais complexos, ou de maior embate físico). Isto é, mulheres mais masculinas em termos de identidade de gênero/sexo gostam mais de esporte. 


\section{Gender/sex identity of athletes and sedentary participants}

ABSTRACT: Are there differences in the process of construction of the gender/sex identity between athletes and non-athletes? This research aimed to compare the self-percept and self-quantified gender/sex identity of athletes from different sport modalities ( 180 men and 180 women) in relation to sedentary participants (22 men and 22 women) regarding to personality and childhood ludic preferences. Men perceived themselves as more masculine and women more feminine. There were more non-heterosexual women than men among the athletes. Men were more likely to enjoy breaking the rules and wanted to be a sports champion when younger. The female athletes who played more with boys in childhood rated themselves as more masculine, daring to transgress the boundaries of gender roles, which may have facilitated their interest in sports training.

KEY-WORDS: Sport; gender identity; masculinity; femininity.

\section{Identidad de género/sexo de atletas y sedentarios}

RESUMEN: ¿Existen diferencias en el proceso de estructuración de la identidad de género/ sexo de atletas y non atletas? Este estudio tuvo como objetivo comparar la identidad de género/sexo auto percibida y auto cuantificada de atletas de diferentes modalidades (180 hombres y 180 mujeres) en relación a sedentarios (22 hombres y 22 mujeres) no tocante a la personalidad e las preferencias ludicas de la infancia. Los hombres perciben a sí mismos más masculinos y las mujeres más femeninas. Se encontró más mujeres no heterosexuales que hombres entre los atletas. Los hombres fueron más propensos a disfrutar de romper las reglas y querían ser un campeón de los deportes cuando niños. Las mujeres que jugaron más con los niños en la infancia se calificaron como más masculinas, atreviéndose a transgredir los límites de los roles de género, lo que puede haber facilitado el interés en el entrenamiento deportivo. PALABRAS-CLAVE: Deporte; identidad de género; masculinidad; feminidad.

\section{REFERÊNCIAS}

ALEXANDER, G. M. An evolutionary perspective of sex-typed toy preferences: pink, blue, and the brain. Archives of Sexual Behavior, New York, v. 32, n. I, p. 7- 14, 2003.

ANDERSON, E. Inclusive masculinity: the changing nature of masculinities. New York: Routledge, 2009.

BAILEY, J. M.; OBERSCHNEIDER, M. Sexual orientation and professional dance. Archives of Sexual Behavior, New York, v. 26, n. 4, p. 433-444, 1997.

BEM, S. L. The measurement of psychological androgyny. Journal of Consulting and Clinical Psychology, Arlington, v. 42, p. I55-162, 1974.

BRADY, K. et al. The role of causal attributions in competitive situations. Sex Roles, New York, v. 35, p. 639-649, 1996. 
BRYCE, J.; RUTTER, J. Digital games and gender. In: BRYCE, J.; RUTTER, J. (Ed.). Understanding digital games. London: Sage, 2006. p. 186-204.

CARDOSO, F. L. O gênero e o movimento humano. Revista Brasileira de Ciências do Esporte, v. 15, n. 3, p. 265-268, 1994.

Conceito de orientação sexual na encruzilhada entre sexo, gênero e motricidade. Interamerican Journal of Psychology, Austin, v. 42, n. 2, p. 69-79, 2008.

. Recalled sex-typed behavior in childhood and sports preferences in adulthood of heterosexual, bisexual, and homosexual men from Brazil, Turkey, and Thailand. Archives of Sexual Behavior, New York, v. 38, p. 726-736, 2009.

et al. Auto-percepção corporal e preferências motoras de praticantes de dança. Movimento, Porto Alegre, v. 16, p. 75-80, 2010.

CHAGAS, E. Educação Física: Escola de formação do corpo feminino. Revista Brasileira de Ciências do Esporte, v. I5, n. 3, p. 226-234, 1994.

COLKER, R.; WIDOM, C. S. Correlates of female athletic participation. Sex Roles, New York, v. 6, p. 47-53, 1980.

COLLEY, A.; ROBERTS, N.; CHIPPS, A. Sex-role identity, personality, and participation in team and individual sports by males and females. International Journal of Sport Psychology, Rome, v. 16, p. 103-112, 1985.

COLWELL, J.; GRADY, C.; RHAKI, S. Computer games, self-esteem and gratification of needs in adolescents. Journal of Community and Applied Social Psychology, Chichester, v. 5, p.195-206, 1995.

CONNOR, J. M.; SERBIN, L. A. Behaviorally based masculine and feminine-activity preferences scales for preschoolers: correlates with other classroom behaviors and cognitive tests. Child Development, Chicago, v. 48, p.|4||-1416, 1977.

COSTA, M. R. F; SILVA, R. G. A educação física e a co-educação: igualdade ou diferença? Revista Brasileira de Ciências do Esporte, Campinas, v. 23, n. 2, p. 43-54, 2002.

CZIMA, K.; WITTIG, A.; SCHURR, K. Sport stereotypes and gender. Journal of Sport and Exercise Psychology, Champaign, v. 10, p. 62-74, 1988.

DAMICO, J. G. S.; MEYER, D. E. O corpo como marcador social: saúde, beleza e valoração de cuidados corporais de jovens mulheres. Revista Brasileira de Ciências do Esporte, v.27, n. 3, p. 103-। 18, 2006.

DONELSON, E.; GULLAHORN, J. Women: a psychological perspective. New York: Willey, 1977.

ECCLES, J. S.; BARBER, B.; JOZEFOWICZ, D.; MALENCHUK, O.; VIDA, M. Self-evaluations of competence, task values, and self-esteem. In: JOHNSON, N.; ROBERTS, M.; WORELL, J. 
(Ed.). Beyond appearance: a new look at adolescent girls. Washington: American Psychological Association, 1999. p. 53-83.

ECCLES, J.; HAROLD, R. Gender differences in sport involvement: Applying the Eccles' expectancy-value model. Journal of Applied Sport Psychology, v. 3, p.7-35, 1991.

GIBBONS, J. L.; LYNN, M. Cross-national gender differences in adolescents' preferences for free-time activities. Cross-Cultural Research, Thousand Oaks, v. 3 I , n. I, p.55-69, 1997.

GILENSTAM, K.; KARP, S.; HENRIKSSON-LARSÉN, K. Gender in ice hockey: women in a male territory. Scandinavian Journal of Medicine \& Science in Sports, v. 18, n. 2, p. 235-249, 2008.

GIULIANO, T. A.; POPP, K. E.; KNIGHT, J. L. Footballs versus Barbies: Childhood play activities as predictors of sport participation by women. Sex Roles, New York, v. 42, n. 3/4, p. |59-18|, 2000.

GREEN, R. The sissy boy syndrome and the development of homosexuality. New Haven: Yale University, 1987.

GREENDORFER, S. L. Gender role stereotypes and early childhood socialization. Psychology of Women Quarterly, New York, v. 18, p. 85-104, 1993.

HARRISON, L. A.; LYNCH, A. B. Social role theory and the perceived gender role orientation of athletes. Sex Roles, New York, v. 52, n. 3/4, p. 227-236, 2005.

HUMBERSTONE, B. Transgressões de gênero e naturezas contestadas. Revista Brasileira de Ciências do Esporte, v. 28, n. 3, p.21-38, 2007.

JAFFEE, L.; MANZER, R. Girls' perspectives: physical activity and self-esteem. Melpomene Journal, v. 3, p. 14-23, 1992.

KAMIZAKI, R. A comparison between the preferences of sporting activities: a psychophysical analysis. Disponível em: http://repository.ispsychophysics.org/fechnerday2007/3/024\%20 Ricardo\%20Kamizaki070822.pdf. Acesso em: I0 jan. 201 I.

KINSEY, A.; POMEROY, W.; MARTIN, C. Sexual behavior in the human male. Philadelphia: Saunders, 1948.

KOCA, C. A.; F. H.; KIRAZCI, S. Gender Role Orientation of Athletes and Nonathletes in a Patriarchal Society: A Study in Turkey. Sex Roles, New York, v. 52, n. 3/4, p. 217-225, 2005.

KOIVULA, N. Rating gender appropriateness of sports participation: Effects of gender-based schematic processing. Sex Roles, New York, v. 33, p. 543-557, 1995.

Sport participation: differences in motivation and actual participation due to gender. Journal of Sport Behavior, v. 22, p. I-22, 1999. 
Perceived characteristics of sports categorized as gender-neutral, feminine and masculine. Journal of Sport Behavior, v. 24, n. 4, p.377-393, 2001.

LAMONT-MILLS, A. The construction of gender identity in elite sport: a mixed methods exploration. Southern Queensland: University of Southern Queensland, 200 I.

LISS, M. B. Patterns of toy play: an analysis of sex differences. Sex Roles, New York, v. 7, p. ||43-||50, | $98 \mid$.

LUCENA, R. F A crônica como gênero que introduziu o esporte no Brasil. Revista Brasileira de Ciências do Esporte, v. 25, n. I, p. I59-171, 2003.

MARTIN, B. A.; MARTIN, J.H. Comparing perceived sex role orientations of the ideal male and female athlete to the ideal male and female person. Journal of Sport Behavior, v. I8, p. 286-301, 1995.

MELO, V. A.; LACERDA, C. Masculinidade, dança e esporte: "Jeux" (Nijinsky, 19|3), "Skating rink" (Borlin, 1922) e "Le train bleu" (Nijinska, 1924). Revista Brasileira de Ciências do Esporte, v. 30, n. 3, p. 45-62, 2009.

MESSNER, M. A.; SABO, D. F. Sex, violence, and power in sports: rethinking masculinity. New York: Crossing Press, 1994

MILLER LITE REPORT ON WOMEN IN SPORTS. East Meadow: Women's Sports Foundation, 1985.

MILLER, J. L.; LEVY, G. D. Gender role conflict, gender-typed characteristics, selfconcepts, and sport socialization in female athletes and nonathletes. Sex Roles, New York, v. 35, p. I I I 122, 1996.

MONEY, J. Gay, straight, and in-between: the sexology of erotic orientation. New York: Oxford University, 1988.

PORMERLEAU, A. et al. Pink or blue: environmental gender stereotypes in the first two years of life. Sex Roles, New York, v. 22, p. 359-367, 1990.

ROMERO, E A. Educação Física a serviço da ideologia sexista. Revista Brasileira de Ciências do Esporte, v. I5, n. 3, p. 226-234, 1994.

RUBENSTEIN, J.; FELDMAN, S. S.; RUBIN, C.; NOVECK, I. A cross-cultural comparison of children's drawings of same-and mixed-sex peer interaction. Journal of Cross-Cultural Psychology, Newbury Park, v. 18, n. 2, p. 234-250, 1987.

SABO, D. F. Sport, patriarchy, and male identity: New questions about men and sport. Arena Review, v. 9, n. 2, p. I-30, 1985.

SARAIVA-KUNZ, M. C. O gênero: confronto de culturas em aulas de Educação Física Revista Brasileira de Ciências do Esporte, v. I5, n. 3, p.226-234, 1994 
SCOTT, J. Gênero uma categoria útil de analise histórica. Educação e Realidade, v. 16, p. 5-22, 1990.

SHAW, S. M.; KLEIBER, D. A.; CALDWELL, L. L. Leisure and identity formation in male and female adolescents: A preliminary examination. Journal of Leisure Research, Washington, $v$. 27, n. 3, p. 245-263, 1995.

SLAVICA J. Differences between boys and girls in terms of physical activities. Facta Universitatis, Nis, v. 8, n. I, p. I-7, 2010.

SNYDER, E. E.; SPREITZER, E. Change and variation in social acceptance of female participation. Journal of Sport Behavior, v.6, p.3-8, 1983.

SOARES, C. L.; GOELLNER, S. V. O elogio a diferença: o avesso da segregação. Revista Brasileira de Ciências do Esporte, v. I 5, n. 3, p. 226-234, 1994.

SOARES, J. P.; ASSIS, Y. A.; SOUSA, F. N. G. Estudo comparativo quanto ao gênero e auto conceito de bailarinos e jogadores de futebol amadores do vale do aço. Revista Movimentum, 2005. Disponível em: <http://www.unilestemg.br/movimentum/index_arquivos>.

TAFFAREL, C. N. Z.; FRANÇA, T. L. A mulher no esporte: o espaço social das praticas esportivas e de produção do conhecimento científico. Revista Brasileira de Ciências do Esporte, v. I5, n.3, p.226-234, 1994.

TOYAMA, J. S. Selected socio-psychological factors as related to the childhood games of successful women. In: KROTEE, M. L. (Ed.). The dimensions of sport sociology. West Point: Leisure, 1977. p.52-59.

WAKEFIELD, J. et al. Androgyny and other measures of masculinity-femininity. Journal of Consulting and Clinical Psychology, Arlington, v. 44, p.766-770, 1976.

WHITAM, F. L. Culturally invariable properties of male homosexuality: tentative conclusions from cross-cultural research. Archives of Sexual Behavior, New York, v. 12, n. 3, p. 207- 226, 1983.

Recebido: I I mar. 20 I I

Aprovado: 3 dez. 2011 Endereço para correspondência:

Fernando Luiz Cardoso.

Rua Arno Hoeschl, I59, apto I 200, centro, Florianópolis, SC, CEP: 880I5-620. Telefone: (48) 332।-8683 ou 99। I-9624 E-mail: fernandocardoso@udesc.brou fernandocardoso.ph.d.lagesc@gmail.com 